\title{
Anatomical Labeling of the Circle of Willis Using Maximum A Posteriori Graph Matching
}

\author{
David Robben ${ }^{1, \star}$, Stefan Sunaert ${ }^{2}$, Vincent Thijs ${ }^{3}$, Guy Wilms ${ }^{2}$, \\ Frederik Maes ${ }^{1}$, and Paul Suetens ${ }^{1}$ \\ 1 iMinds - Medical Image Computing (ESAT/PSI), KU Leuven, Belgium \\ 2 Department of Radiology, University Hospitals Leuven, KU Leuven, Belgium \\ 3 Department of Neurology, University Hospitals Leuven, KU Leuven, Belgium \\ david.robben@esat.kuleuven.be
}

\begin{abstract}
A new method for anatomically labeling the vasculature is presented and applied to the Circle of Willis. Our method converts the segmented vasculature into a graph that is matched with an annotated graph atlas in a maximum a posteriori (MAP) way. The MAP matching is formulated as a quadratic binary programming problem which can be solved efficiently. Unlike previous methods, our approach can handle non tree-like vasculature and large topological differences. The method is evaluated in a leave-one-out test on MRA of 30 subjects where it achieves a sensitivity of $93 \%$ and a specificity of $85 \%$ with an average error of 1.5 $\mathrm{mm}$ on matching bifurcations in the vascular graph.
\end{abstract}

Keywords: Graph matching, vasculature, anatomical labeling, Circle of Willis, MAP.

\section{Introduction}

The Circle of Willis (CoW) is a circle of arteries in the skull base that connects the left and right side of the anterior cerebral circulation with the posterior cerebral circulation. This structure is highly variable: only $42 \%$ of the population has a complete circle [2] and in the other cases, one or more arteries are missing. In the past years, several research papers addressed the problem of segmenting the $\mathrm{CoW}$ and the cerebral vasculature in general, without labeling them. Having an algorithm that can anatomically label the vasculature, however, can be important for many applications. In clinical settings, it can give an interventional radiologist additional guidance when navigating through the vasculature of a patient, or it can provide automatic measurements of the diameter of certain vessels. In a research context, it can be used to detect patterns in the vasculature of subpopulations (e.g. geometric risk factors for vascular pathologies) in a more accurate fashion. Despite these applications, literature is very limited. Since a similar problem is encountered in the labeling of the bronchial segments in the lungs, we also consider this in our literature study.

\footnotetext{
* David Robben is supported by a Ph.D. fellowship of the Research Foundation Flanders (FWO).
} 
Most methods first transform the segmented image into a graph, and then label this graph. Tschirren et al. 9] use atlas-based graph matching to label the bronchi of a patient. Although a probabilistic graph atlas is created - containing the average and standard deviation of certain properties - the matching has no probabilistic interpretation. Mori et al. published several works about bronchial labeling. Their latest approach [6] uses multiclass AdaBoost to assign a probability to each branch and label. Subsequently, a depth-first search finds the global optimal assignment of branch names taking into account topological constraints. In another line of work, Mori et al. [5] label the abdominal arteries, which they consider more difficult than labeling bronchi due to the larger variation. They state that their approach is expected not to work on vasculature of other organs. Bogunovic et al. 1] label 5 bifurcations in the anterior circulation of the CoW. They are the first to introduce a maximum a posteriori approach to the matching problem. All possible isomorphisms between the target graph and the atlas are explicitly enumerated and for each mapping the likelihood is calculated to find the maximum. The likelihood term only compares the bifurcations but not the branches in between. Although the performance of the method is very good, it is not computationally scalable: a preprocessing step is required to prune the graph to about 20 candidate vertices. It should be noted that all of the previous methods assume that the graph is a tree and allow limited or even no topological variations.

Takemura et al. 8] propose a method that does not use graphs. They label the CoW by rigidly registering the to-be-labeled image to an atlas. Due to the large variability in the shape of the vasculature, the quality of results varies enormously between the different subjects and for the different arteries. They report voxel-wise classification rates as high as $86 \%$ for large vessels as the Internal Carotid Artery (ICA) and as low as $0.5 \%$ for small vessels such as the Posterior Communicating Artery (PcomA).

This paper introduces a novel and generic approach to anatomically label vessels, and demonstrates its performance on the CoW and its adjacent vessels. In this paper, we label 24 Points of Interest (PoI): 15 bifurcations and 9 endpoints of the cerebral vasculature (Fig. 1).

\section{Methodology}

\subsection{Creation of a Vascular Graph}

The first step in our algorithm is the conversion of a vascular image into an attributed graph $G=\left(V_{G}, E_{G}, A_{G}\right)$ with $V_{G}$ the set of vertices, $E_{G}$ the set of edges and $A_{G}$ the attributes of the vertices and edges. The vertices $V_{G}$ represent the bifurcations and endpoints of the vessels and the edges $E_{G}$ represent the vascular trajectories between the - both directly and indirectly connected - vertices. In case multiple paths connect two points, the shortest is used to calculate the attributes. Segmentation of the MRA images is done with region growing and this segmented image is subsequently thinned using Palagyi's thinning algorithm [7. The resulting skeleton image is converted into a graph and 


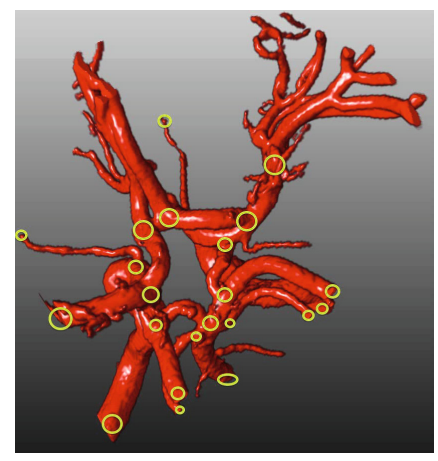

(a) Complete CoW.

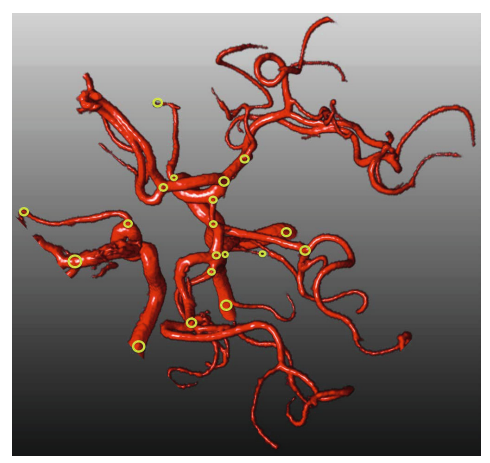

(b) Missing first segment of the Anterior Cerebral Artery (ACA1) and missing PcomA.

Fig. 1. Two (clipped views of the) CoW and our points of interest

the attributes are calculated. The attributes of an edge $e_{G}=\left(v_{G}, v_{G}^{\prime}\right)$ are: the Euclidean and geodesic length, the average radius and the relative position of $v_{G}$ to $v_{G}^{\prime}$. The only attributes for the vertices are the position and a rotation invariant bifurcation descriptor which is a vector that contains the fraction of vessel-to-background on a sphere centered around the vertex for six different radii.

\subsection{Atlas Construction}

The creation of an atlas requires a training set of vascular graphs and the ground truth annotation of the PoI on these graphs. Note that not every PoI will be present in each vascular graph. The atlas itself is a probabilistic attributed graph $A=\left(V_{A}, E_{A}, A_{A}\right)$. The vertices $V_{A}$ are the PoIs, the edges $E_{A}$ the relations between those PoIs and $A_{A}$ the attributes. The attributes of a vertex $v_{A}$ are: the probability that this point exists in an image $P_{\text {exist }, v}$ and the probability distribution of its position and feature vector. The attributes of an edge $e_{A}=\left(v_{A}, v_{A}^{\prime}\right)$ are: the probability of existence of a branch between $v_{A}$ and $v_{A}^{\prime}$, called $P_{\text {exist,e }}$, the probability distribution of its Euclidean length $P_{l E}$, geodesic length $P_{l}$ and radius $P_{r}$, the probability distribution of the relative position of $v_{A}$ to $v_{A}^{\prime}$, called $P_{\Delta x y z}$, and the probability that another PoI $v_{A}^{\prime \prime}$ lies on this edge $P_{\text {on }\left(v_{A}, v_{A}^{\prime}\right)}\left(v_{A}^{\prime \prime}\right)$. The atlas also contains the prior distribution for all these attributes, that is the distribution taken over all vertices or edges in the images. All probability distributions are calculated using one-dimensional kernel density estimation.

\subsection{MAP Graph Matching}

Labeling an image corresponds to finding a match between the vascular graph $G$ and the atlas $A$ (see Fig. 2). The match $M$ is a subset of the candidate 


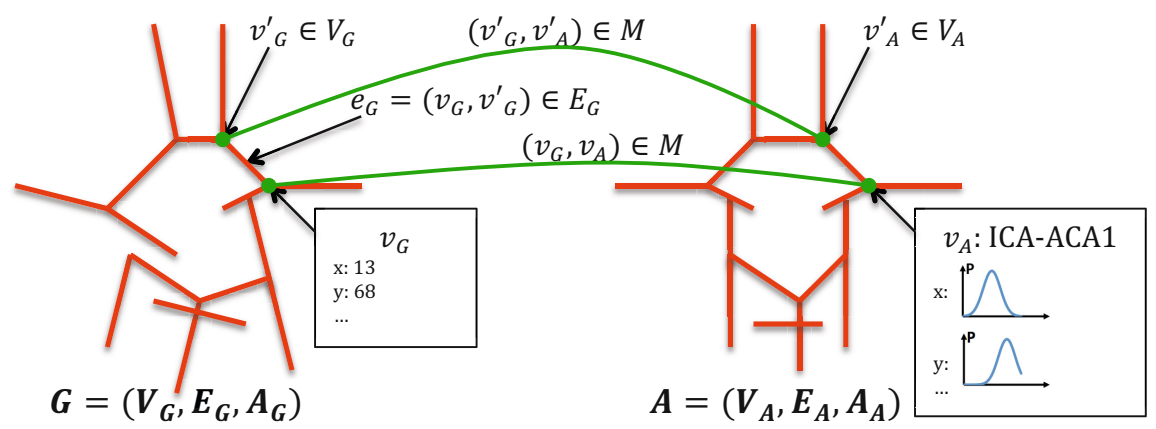

Fig. 2. The terminology used in graph matching

correspondences $C C=\left\{\left(v_{G}, v_{A}\right) \mid v_{G} \in V_{G}, v_{A} \in V_{A}\right\}$, with the additional constraint that every vertex $v_{A}$ or $v_{G}$ can only occur once in the match $M$. We are interested in the match $M^{*}$ with the maximum a posteriori probability:

$$
M^{*}=\operatorname{argmax}_{M} P(M \mid G, A)=\operatorname{argmax}_{M} P(G \mid M, A) P(M \mid A) .
$$

We derive a general formulation to efficiently solve this problem taking into account information about both the bifurcations and the edges.

To simplify the notation, we introduce two additional sets. The set of vertices of $A$ that occur in match $M$ will be called $V_{A, M}=\left\{v_{A} \mid\left(v_{G}, v_{A}\right) \in M\right\}$ and the set of vertices of $A$ that do not occur in match $M$ will be called $V_{A, \bar{M}}=\left\{v_{A} \mid v_{A} \in\right.$ $\left.V_{A}, v_{A} \notin V_{A, M}\right\}$. The set of vertices of $G$ that occur in match $M$ will be called $V_{G, M}=\left\{v_{G} \mid\left(v_{G}, v_{A}\right) \in M\right\}$ and the set of vertices of $G$ that do not occur in match $M$ will be called $V_{G, \bar{M}}=\left\{v_{G} \mid v_{G} \in V_{G}, v_{G} \notin V_{G, M}\right\}$.

Matching Prior. The prior term $P(M \mid A)$ states how likely a certain match a priori is: not every PoI has an equal probability to exist in $G$ and thus to be matched. Assuming independency:

$$
P(M \mid A)=\prod_{v_{A} \in V_{A, M}} P_{e x i s t, v}\left(v_{A}\right) \cdot \prod_{v_{A} \in V_{A, \bar{M}}}\left(1-P_{\text {exist }, v}\left(v_{A}\right)\right)
$$

where $P_{\text {exist }}\left(v_{A}\right)$ is the probability that $v_{A}$ is present in an image. By taking the logarithm, this term can be rewritten as a sum of a constant term and a term depending on $M$ :

$$
\log P(M \mid A)=\sum_{v_{A} \in V_{A}} \log \left(1-P_{\text {exist }, v}\left(v_{A}\right)\right)+\sum_{v_{A} \in V_{A}, M} \log \frac{P_{\text {exist }, v}\left(v_{A}\right)}{1-P_{\text {exist }, v}\left(v_{A}\right)} .
$$


Matching Likelihood. The likelihood term $P(G \mid M, A)$ expresses the probability that graph $G$ exists, given the atlas and the match:

$$
\begin{aligned}
P(G \mid M, A)= & \prod_{\left(v_{G}, v_{A}\right) \in M}\left[P_{a t t}\left(v_{G} \mid v_{A}\right) \cdot \prod_{\left(v_{G}^{\prime}, v_{A}^{\prime}\right) \in M} \sqrt{P_{a t t}\left(\left(v_{G}, v_{G}^{\prime}\right) \mid\left(v_{A}, v_{A}^{\prime}\right)\right)}\right. \\
\cdot \prod_{v_{G}^{\prime} \in V_{G, \bar{M}}} & \left.\sqrt{P_{a t t}\left(\left(v_{G}, v_{G}^{\prime}\right)\right)}\right] \\
\cdot \prod_{v_{G} \in V_{G, \bar{M}}}\left[P_{a t t}\left(v_{G}\right) \cdot \prod_{v_{G}^{\prime} \in V_{G, \bar{M}}}\right. & \left.\sqrt{P_{a t t}\left(\left(v_{G}, v_{G}^{\prime}\right)\right)}\right] .
\end{aligned}
$$

In this equation $P_{a t t}\left(v_{G} \mid v_{A}\right)$ is the probability that $v_{A}$ has the attributes of $v_{G}$, $P_{a t t}\left(\left(v_{G}, v_{G}^{\prime}\right) \mid\left(v_{A}, v_{A}^{\prime}\right)\right)$ is the probability that the edge between $v_{A}$ and $v_{A}^{\prime}$ has the attributes of the edge between $v_{G}$ and $v_{G}^{\prime}, P_{a t t}\left(v_{G}\right)$ is the prior probability that a vertex has the attributes of $v_{G}$ and $P_{a t t}\left(\left(v_{G}, v_{G}^{\prime}\right)\right)$ is the prior probability that an edge has the attributes of the edge between $v_{G}$ and $v_{G}^{\prime}$. Table 1 states how these terms are calculated for our chosen attributes. By taking the logarithm, the likelihood can be rewritten as a sum of a constant term and a term depending on $M$ :

$$
\begin{aligned}
& \log P(G \mid M, A)=\sum_{v_{G} \in V_{G}}\left[\log P_{a t t}\left(v_{G}\right)+\frac{1}{2} \sum_{v_{G}^{\prime} \in V_{G}} \log P_{a t t}\left(\left(v_{G}, v_{G}^{\prime}\right)\right)\right] \\
& +\sum_{\left(v_{G}, v_{A}\right) \in M}\left[\log \frac{P_{a t t}\left(v_{G} \mid v_{A}\right)}{P_{a t t}\left(v_{G}\right)}+\frac{1}{2} \sum_{\left(v_{G}^{\prime}, v_{A}^{\prime}\right) \in M} \log \frac{P_{a t t}\left(\left(v_{G}, v_{G}^{\prime}\right) \mid\left(v_{A}, v_{A}^{\prime}\right)\right)}{P_{a t t}\left(\left(v_{G}, v_{G}^{\prime}\right)\right)}\right]
\end{aligned}
$$

Quadratic Binary Programming. Now we introduce $x$, a binary vector of length $|C C|$ that is equivalent to $M$ by $x[i]==1 \Leftrightarrow C C[i] \in M$. The MAP problem can be written as a Quadratic Binary Programming problem $x^{T} Q x . Q$ is a symmetric matrix of size $|C C| \times|C C|$ with its elements determined by the previous equations:

$$
Q_{i, i}=\log \frac{P_{\text {exist }, v}\left(v_{A}\right)}{1-P_{\text {exist }, v}\left(v_{A}\right)}+\log \frac{P_{a t t}\left(v_{G} \mid v_{A}\right)}{P_{a t t}\left(v_{G}\right)} \quad Q_{i, j}=\frac{1}{2} \log \frac{P_{a t t}\left(\left(v_{G}, v_{G}^{\prime}\right) \mid\left(v_{A}, v_{A}^{\prime}\right)\right)}{P_{a t t}\left(\left(v_{G}, v_{G}^{\prime}\right)\right)}
$$

with $\left(v_{G}, v_{A}\right)=C C[i]$ and $\left(v_{G}^{\prime}, v_{A}^{\prime}\right)=C C[j]$. Additionally, to prevent double assignment of a certain label, if $C C[k]$ and $C C[l]$ contain the same graph or atlas vertex, $Q_{k, l}=-\infty$. The a posteriori probability is (up to a constant) equivalent to $x^{T} Q x$ and thus: $M^{*} \equiv x^{*}=\operatorname{argmax}_{x} x^{T} Q x$.

\subsection{Matching}

Once $Q$ is formed, matching is equivalent to finding the binary vector $x^{*}$ with highest score $x^{T} Q x$. The largest eigenvector of $Q$ serves as an initialization 3 and randomized greedy search [4] optimizes the result further. Then we check for every three found correspondences $\left(v_{G}, v_{A}\right),\left(v_{G}^{\prime}, v_{A}^{\prime}\right),\left(v_{G}^{\prime \prime}, v_{A}^{\prime \prime}\right)$ if the connectivity 
Table 1. Example of how $P_{a t t}$ is defined, with $e_{G}=\left(v_{G}, v_{G}^{\prime}\right)$ and $e_{A}=\left(v_{A}, v_{A}^{\prime}\right)$. The posterior distribution is described in the first column, the prior distribution in the second column. The probability calculation depends on the connectivity in $G$ : if $v_{G}$ and $v_{G}^{\prime}$ are connected, the first row is considered, otherwise the second.

\begin{tabular}{|c|c|c|}
\hline & $\mid P_{a t t}\left(e_{G} \mid e_{A}\right)$ & $P_{a t t}\left(e_{G}\right)$ \\
\hline$\left(v_{G}, v_{G}^{\prime}\right) \in E_{G}$ & $\begin{array}{l}P_{\text {exist }, e}\left(e_{G} \mid e_{A}\right) \\
\quad \cdot P_{r}\left(e_{G} \mid e_{A}\right) \cdot P_{l}\left(e_{G} \mid e_{A}\right) \\
\quad . P_{l E}\left(e_{G} \mid e_{A}\right) \cdot P_{\Delta x y z}\left(e_{G} \mid e_{A}\right) \\
\end{array}$ & $\begin{array}{l}P_{\text {exist }, e}\left(e_{G}\right) \\
\quad . P_{r}\left(e_{G}\right) \cdot P_{l}\left(e_{G}\right) \\
\quad . P_{l E}\left(e_{G}\right) \cdot P_{\Delta x y z}\left(e_{G}\right) \\
\end{array}$ \\
\hline$\left(v_{G}, v_{G}^{\prime}\right) \notin E_{G}$ & $\begin{array}{l}\left(1-P_{\text {exist }, e}\left(e_{G} \mid e_{A}\right)\right) \\
\quad \cdot P_{l E}\left(e_{G} \mid e_{A}\right) \cdot P_{\Delta x y z}\left(e_{G} \mid e_{A}\right)\end{array}$ & $\begin{array}{l}\left(1-P_{\text {exist }, e}\left(e_{G}\right)\right) \\
\quad P_{l E}\left(e_{G}\right) \cdot P_{\Delta x y z}\left(e_{G}\right)\end{array}$ \\
\hline
\end{tabular}

of $v_{G}, v_{G}^{\prime}$ and $v_{G}^{\prime \prime}$ in $G$ is allowed according to $P_{o n}\left(v_{A}, v_{A}^{\prime}\right)\left(v_{A}^{\prime \prime}\right)$. The most violating correspondence is removed from $x, C C$ and $Q$ and the search continues. This process is repeated until there are no more violations.

\section{Evaluation}

The evaluation used a dataset of 30 TOF-MRA images acquired on three different scanners (Philips Intera, Philips Ingenia and Philips Achieva). Both males and females are included and the age varies between 20 and 82 . The dataset contains both healthy and diseased vasculature, with the latter having stenosis or aneurisms. Six topological variants of the $\mathrm{CoW}$ are present: there are $\mathrm{CoW}$ with the Anterior Communicating Artery (AComA), the left and/or right PComA and/or the right ACA1 missing. The voxel size is $0.39 \times 0.39 \times 0.5 \mathrm{~mm}^{3}$. All images were segmented using region growing and converted to annotated vascular graphs. On average, one vascular graph contains 197 vertices. An observer (D.R.) manually indicated the Points of Interest (PoI) on each vascular graph, which serves as ground truth. In total a maximum of 30 images $\times 24 \mathrm{PoI} /$ image $=720$ PoI could be present in those 30 images, but only 575 PoI were present.

A leave-one-out cross-validation was performed to asses the performance of the proposed method. This means that every image was matched once, using an atlas that is constructed using the other 29 images. The construction of an atlas based on the annotated vascular graphs takes about 5 minutes using single-threaded Python code on an Intel Core i5 at 2.7 Ghz. The running time for matching a graph with the atlas depends on $|C C|$, the number of candidate correspondences considered. Using $C C=\left\{\left(v_{G}, v_{A}\right) \mid P_{a t t}\left(v_{G} \mid v_{A}\right)>0\right\}$, on average 1000 candidate correspondences are considered and matching requires 2 minutes running time on the same computer. Every matching succeeded and the results are summarized in Fig. 3. In total, there are 540 true positives with an average error of $1.5 \mathrm{~mm}$, 21 false positives (PoI that do not exist in the image but are assigned), 124 true negatives and 35 false negatives (PoI that are in the image but are not assigned). Two labeled images are shown in Fig. 4. We notice that the large majority of the correspondences is correct, and the label is placed on the exact same place as in the ground truth. The larger errors are without exception caused by the PoI that 


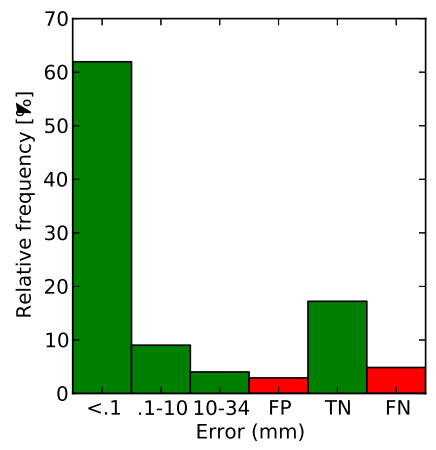

\begin{tabular}{l|r|r|r|r|r} 
PoI & TP & \% FP & \% $\mathrm{TN}$ & \% FN & error $(\mathrm{mm})$ \\
\hline ICA-OA & 65 & 17 & 13 & 5 & 0.40 \\
PCA2 End & 93 & 0 & 3 & 3 & 1.78 \\
PCA1-PComA & 7 & 0 & 82 & 12 & 0.00 \\
ACA1-AComA & 87 & 8 & 2 & 3 & 0.99 \\
VBA-PCA1 & 97 & 0 & 0 & 3 & 0.46 \\
VBA End & 100 & 0 & 0 & 0 & 1.31 \\
ICA End & 100 & 0 & 0 & 0 & 0.94 \\
M1-M2 & 95 & 0 & 0 & 5 & 3.72 \\
ICA-PCoA & 10 & 0 & 82 & 8 & 0.00 \\
ICA-ACA1 & 88 & 2 & 2 & 8 & 0.65 \\
SCA End & 98 & 0 & 0 & 2 & 4.45 \\
OA End & 60 & 8 & 23 & 8 & 0.21 \\
VBA-SCA & 98 & 0 & 0 & 2 & 0.35
\end{tabular}

(a) Error distribution for all PoI. (b) Results for each PoI (left and right combined). Error is the average error of the TP.

Fig. 3. Results of the leave-one-out test
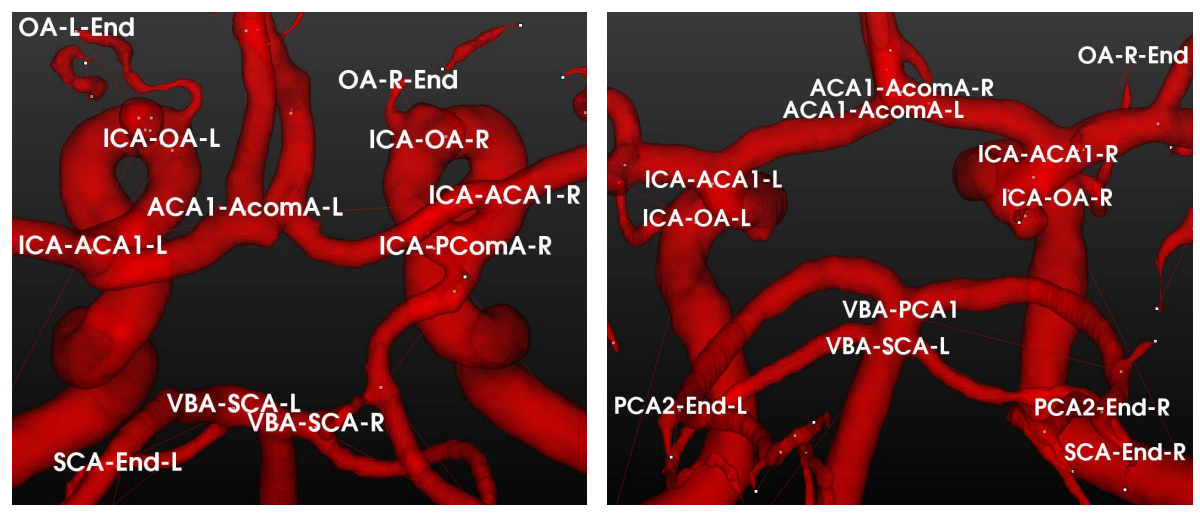

Fig. 4. Two automatically labeled images

are endpoints in the atlas: these points are directly connected to only one other PoI and are thus less well defined than PoI that are connected to three other PoI. If the endpoints are not considered, the maximum error is $12 \mathrm{~mm}$ and the average error $0.6 \mathrm{~mm}$. Usage of better feature vectors for the bifurcations should probably minimize this problem, as was demonstrated by Bogunovic [1]. For 26 out of the 30 matchings, the score $x^{* T} Q x^{*}$ is greater than the ground truth score, which also suggests that better features are the key to better labeling.

\section{Conclusion}

A new vascular labeling algorithm is introduced. To our best knowledge it is the first graph-based algorithm that does not require a tree structure of the vasculature. As such, it is capable to label the complete Circle of Willis. The 
problem is formulated as a maximum a posteriori formulation that can be solved efficiently using Quadratic Binary Programming. The approach was evaluated in a leave-one-out test on 30 MRA images. In future work, we will use better feature vectors for the bifurcations. Additionally, we expect that a rigid registration of the vascular images prior to creation of the vascular graphs will result in an atlas with more sharply defined probability distributions for the attributes and thus better matching. We also intend to enlarge the dataset to support even more variations of the CoW. Finally, although the segmentation itself is good, the thinning is not optimal as kissing vessels can form spurious, topology changing bifurcations that cause additional variability and make labeling more challenging. We are exploring centerline segmentation algorithms and combined segmentation/labeling to alleviate this problem.

\section{References}

1. Bogunović, H., Pozo, J.M., Cárdenes, R., Frangi, A.F.: Anatomical labeling of the anterior circulation of the Circle of Willis using maximum a posteriori classification. In: Fichtinger, G., Martel, A., Peters, T. (eds.) MICCAI 2011, Part III. LNCS, vol. 6893, pp. 330-337. Springer, Heidelberg (2011)

2. Krabbe-Hartkamp, M., Van der Grond, J., de Leeuw, F., de Groot, J., Algra, A., Hillen, B., Breteler, M., Mali, W.: Circle of Willis; morphologic variation on threedimensional time-of-flight MR angiograms. Radiology 207(1), 103-111 (1998)

3. Leordeanu, M., Hebert, M.: A spectral technique for correspondence problems using pairwise constraints. In: ICCV, vol. 2, pp. 1482-1489 (2005),

http://www.ri.cmu.edu/publication_view.html?pub_id=5161

4. Merz, P., Freisleben, B.: Greedy and local search heuristics for unconstrained binary quadratic programming. Journal of Heuristics 8(2), 197-213 (2002)

5. Mori, K., Oda, M., Egusa, T., Jiang, Z., Kitasaka, T., Fujiwara, M., Misawa, K.: Automated nomenclature of upper abdominal arteries for displaying anatomical names on virtual laparoscopic images. In: Liao, H., Edwards, P.J., Pan, X., Fan, Y., Yang, G.-Z. (eds.) MIAR 2010. LNCS, vol. 6326, pp. 353-362. Springer, Heidelberg (2010)

6. Mori, K., Ota, S., Deguchi, D., Kitasaka, T., Suenaga, Y., Iwano, S., Hasegawa, Y., Takabatake, H., Mori, M., Natori, H.: Automated anatomical labeling of bronchial branches extracted from CT datasets based on machine learning and combination optimization and its application to bronchoscope guidance. In: Yang, G.-Z., Hawkes, D., Rueckert, D., Noble, A., Taylor, C. (eds.) MICCAI 2009, Part II. LNCS, vol. 5762, pp. 707-714. Springer, Heidelberg (2009)

7. Palágyi, K., Sorantin, E., Balogh, E., Kuba, A., Halmai, C., Erdohelyi, B., Hausegger, K.: A sequential 3D thinning algorithm and its medical applications. In: Insana, M.F., Leahy, R.M. (eds.) IPMI 2001. LNCS, vol. 2082, pp. 409-415. Springer, Heidelberg (2001)

8. Takemura, A., Suzuki, M., Harauchi, H., Okumura, Y.: Automatic anatomical labeling method of cerebral arteries in MR-angiography data set. Japanese Journal of Medical Physics 26(4), 187-198 (2006)

9. Tschirren, J., McLennan, G., Palágyi, K., Hoffman, E.A., Sonka, M.: Matching and anatomical labeling of human airway tree. IEEE Transactions on Medical Imaging 24(12), 1540-1547 (2005) 